\title{
ON THE HOPF-ZERO BIFURCATION OF THE MICHELSON SYSTEM
}

\author{
JAUME LLIBRE ${ }^{1}$ AND XIANG ZHANG ${ }^{2}$
}

\begin{abstract}
Applying a new result for studying the periodic orbits of a differential system via the averaging theory, we provide the first analytic proof on the existence of a Hopf-zero bifurcation for the Michelson system

$$
\dot{x}=y, \quad \dot{y}=z, \quad \dot{z}=c^{2}-y-\frac{x^{2}}{2},
$$

at $c=0$. Moreover our method estimates the shape of this periodic orbit in function of $c>0$ sufficiently small.
\end{abstract}

\section{InTRODUCTION AND STATEMENT OF RESULTS}

The Michelson system

$$
\dot{x}=y, \quad \dot{y}=z, \quad \dot{z}=c^{2}-y-\frac{x^{2}}{2},
$$

with $(x, y, z) \in \mathbb{R}^{3}$ and the parameter $c \geq 0$, was obtained by Michelson [8] in the study of the travelling wave solutions of the Kuramoto-Sivashinsky equation. It is well known that system (1) is reversible with respect to the involution $R(x, y, z)=(-x, y,-z)$ and is volume-preserving under the flow of the system. It is easy to check that system (1) has two finite singularities $S_{1}=(-\sqrt{2} c, 0,0)$ and $S_{2}=(\sqrt{2} c, 0,0)$ for $c>0$, which are both saddlefoci. The former has a 2-dimensional stable manifold and the latter has a 2-dimensional unstable manifold.

In what follows we do a brief summary on the dynamics results on system (1). The first study on system (1) goes back to Michelson [8] who proved that if $c>0$ is sufficiently large, system (1) has a unique bounded solution, which is the transversal heteroclinic orbit connecting the two finite singularities. When $c$ decreases there will appears the cocoon bifurcation, which was verified in $[8,7,4]$ using computer assistance. As for the appearance of the cocoon bifurcation, Remark 1.6 of [2] explained that if there exists a saddle-node periodic orbit $\gamma$ at some value $c_{0}>0$ of the parameter which is symmetric with respect to the involution $R$, then for $c$ on the one side of $c_{0}$ the saddle-node periodic orbits split into two limit cycles, while for $c$

2000 Mathematics Subject Classification. 34C05, 34C23, 34C25, 34C29.

Key words and phrases. Michelson system, periodic orbit, averaging method. 
on the other side of $c_{0}$ no periodic orbits will be present near $\gamma$, but a cocooning cascade appears. Lamb et al [6] proved that the Michelson system can have a countable infinitely many heteroclinic loops, a countable infinitely many homoclinic loops, and a countable infinity of hyperbolic base sets (horseshoes).

For $c>0$ small numerical experiments (see for instance Kent and Elgin [3]) and asymptotic expansions in sinus series (see Michelson [8] in 1986 and Webster and Elgin [11] in 2003) revealed the existence of a Hopf-zero bifurcation at the origin for $c=0$. But their results do not provide an analytic proof on the existence of such Hopf-zero bifurcation. By a Hopfzero bifurcation we mean that when $c=0$ the Michelson system has the origin as a singularity having eigenvalues $0, \pm i$, and when $c>0$ sufficiently small the Michelson system has a periodic orbit which tends to the origin when $c$ tends to zero. For completeness in the next section we will summarize the results on the periodic orbits of the Michelson system.

The main goal of this paper is to provide such an analytic proof. The tool is a new developed averaging method, see [1].

Theorem 1. For $c \geq 0$ sufficiently small the Michelson system (1) has a Hopf-zero bifurcation at the origin for $c=0$. Moreover the bifurcated periodic orbit satisfies $x(t)=-2 c \cos t+o(c), y(t)=2 c \sin t+o(c)$ and $z(t)=2 c \cot t+o(c)$ for $c>0$ sufficiently small.

The proof of Theorem 1 is given in Section 3 .

\section{Summary OF KNOWN RESUlts ON PERIODIC ORBITS}

The first asymptotic result on the existence of periodic orbits of system (1) is given by Michelson [8] using a series in sinus for small $c$, and it can be stated as:

Remark 2. For $c>0$ small the Michelson system has a periodic orbit whose approximation by a sinus series is

$$
x(t)=2 c \sin \Omega t-\frac{c^{2}}{6} \sin 2 \Omega t+\frac{c^{3}}{144} \sin 3 \Omega t+\ldots,
$$

where $\Omega=1-c^{2} / 12+\cdots$.

We remark that the periodic orbit in Theorem 2 is also founded approximately by Liapunov-Schmidt reduction method [6].

Using the periodic orbit (2) as a starting point for the continuation code AUTO, Kent and Elgin [3] obtained numerically the so-called noose bifurcation, i.e. in an ordinary differential system depending on a parameter $\lambda$ there is a periodic solution which has a period-doubling at $\lambda=\lambda_{1}$, then the two branches emerging from this periodic orbit come together and annihilate 
in a saddle-node periodic orbit at some other value $\lambda=\lambda_{2}$. In short we can say:

Remark 3. The Michelson system can have a noose bifurcation initiated in a periodic orbit coming from the Hopf-zero bifurcation at the origin at $c=0$.

For the existence of more than one periodic orbits Troy [9] proved the following result.

Theorem 4. For $c=1$ the Michelson system has at least two periodic solutions, which are odd in $t$.

Kent comments that these periodic orbits are symmetric with respect to the involution $R$ (see [10] page 36). Recall from [5] that a symmetric periodic orbit with respect to the involution $R$ must intersect with $\operatorname{Fix}(R)$ (the set of fixed point of $R$ ), i.e. the $y$-axis.

The following result, due to Lamb et al [6], shows that the Michelson system can have infinitely many symmetric periodic orbits with respect to the involution $R$.

Theorem 5. For each $n \in \mathbb{N}$ there exist values of the parameter $c>0$ for which the Michelson system can have a countable infinity of n-periodic orbits accumulating to $n$-heteroclinic and $n$-homoclinic cycles which connect the two singularities $S_{1}$ and $S_{2}$.

The following result, due to Kokubu et al [4], presents the appearance of saddle-node periodic orbit.

Theorem 6. There exists a value $c_{\infty}$ for which the following hold.

(a) The Michelson system has a saddle-node periodic orbit $\gamma_{*}$ which is symmetric under the involution $R$. Here by a saddle-node periodic orbit we mean a periodic orbit whose Poincaré map has both eigenvalues equal to unity.

(b) The saddle-node periodic orbit $\gamma_{*}$ is generic and generically unfolded under the reversibility with respect to $R$. Here the genericity means that some of the derivatives of the Poincaré map at the saddle-node periodic orbit are nonzero.

(c) $W^{u}\left(\gamma_{*}\right)$ and $W^{s}\left(S_{1}\right)$, as well as $W^{s}\left(\gamma_{*}\right)$ and $W^{u}\left(S_{2}\right)$, intersect transversally, where $W^{u}(\alpha)$ and $W^{s}(\alpha)$ with $\alpha \in\left\{\gamma_{*}, S_{1}, S_{2}\right\}$ are the unstable and stable manifolds of $\alpha$ respectively. 


\section{Proof of Theorem 1}

The following result, due to Buică, Françoise and Llibre [1], will be used in the proof of Theorem 1.

Theorem 7. (Perturbations of an isochronous set) Consider a differential system

(3) $\dot{x}=F_{0}(t, x)+\varepsilon F_{1}(t, x)+\varepsilon^{2} F_{2}(t, x, \varepsilon), \quad(t, x, \varepsilon) \in \mathbb{R} \times \Omega \times\left(-\varepsilon_{0}, \varepsilon_{0}\right)$,

where $\Omega$ is an open subset of $\mathbb{R}^{n}$, and $F_{0}, F_{1}$ and $F_{2}$ are $C^{2}$ smooth and $T$-periodic in the time $t$. Let $x(t, z)$ be a solution of $(3)$ when $\varepsilon=0$ such that $x(0, z)=z$. Denote by $M_{z}(t)$ the fundamental solution matrix of the variational equation

$$
\dot{y}=D_{x} F_{0}(t, x(t, z)) y,
$$

such that $M_{z}(0)=$ identity. Assume that there exists an open and bounded subset $V$ with its closure $\operatorname{cl}(V) \subset \Omega$ such that for each $z \in \operatorname{cl}(V)$, the solution $x(t, z)$ is T-periodic. If $a \in V$ is a zero of the map $\mathcal{F}: \operatorname{cl}(V) \rightarrow \mathbb{R}^{n}$ defined by

$$
\mathcal{F}(z)=\int_{0}^{T} M_{z}^{-1}(t) F_{1}(t, x(t, z)) d t,
$$

and $\operatorname{det}\left(D_{z} \mathcal{F}(a)\right) \neq 0$, then for $|\varepsilon|>0$ sufficiently small system (3) has a $T$-periodic solution $\phi(t, \varepsilon)$ such that $\phi(0, \varepsilon) \rightarrow a$ as $\varepsilon \rightarrow 0$.

Proof of Theorem 1: For any $\varepsilon \neq 0$ we take the change of variables $x=$ $\varepsilon \bar{x}, y=\varepsilon \bar{y}, z=\varepsilon \bar{z}$ and $c=\varepsilon d$, then the Michelson system (1) becomes

$$
\dot{x}=y, \quad \dot{y}=z, \quad \dot{z}=-y+\varepsilon d^{2}-\varepsilon \frac{x^{2}}{2},
$$

where we still use $x, y, z$ instead of $\bar{x}, \bar{y}, \bar{z}$. Now doing the change of variables $x=x, y=r \sin \theta$ and $z=r \cos \theta$, system (5) goes over to

(6) $\quad \dot{x}=r \sin \theta, \quad \dot{r}=\frac{\varepsilon}{2}\left(2 d^{2}-x^{2}\right) \cos \theta, \quad \dot{\theta}=1-\frac{\varepsilon}{2 r}\left(2 d^{2}-x^{2}\right) \sin \theta$.

This system can be written as

$$
\begin{aligned}
& \frac{d x}{d \theta}=r \sin \theta+\frac{\varepsilon}{2}\left(2 d^{2}-x^{2}\right) \sin ^{2} \theta+\varepsilon^{2} f_{1}(\theta, r, \varepsilon), \\
& \frac{d r}{d \theta}=\frac{\varepsilon}{2}\left(2 d^{2}-x^{2}\right) \cos \theta+\varepsilon^{2} f_{2}(\theta, r, \varepsilon),
\end{aligned}
$$

where $f_{1}$ and $f_{2}$ are analytic functions in their variables.

For any given $x_{0}$ and $r_{0}$, system $(7)_{\varepsilon=0}$ has the $2 \pi$-periodic solution

$$
x(\theta)=r_{0}+x_{0}-r_{0} \cos \theta, \quad r(\theta)=r_{0},
$$


such that $x(0)=x_{0}$ and $r(0)=r_{0}$. It is easy to see that the variational equation of $(7)_{\varepsilon=0}$ along the solution (8) is

$$
\left(\begin{array}{l}
\frac{d y_{1}}{d \theta} \\
\frac{d y_{2}}{d \theta}
\end{array}\right)=\left(\begin{array}{cc}
0 & \sin \theta \\
0 & 0
\end{array}\right)\left(\begin{array}{l}
y_{1} \\
y_{2}
\end{array}\right)
$$

It has the fundamental solution matrix

$$
M=\left(\begin{array}{cc}
1 & 1-\cos \theta \\
0 & 1
\end{array}\right),
$$

which is independent of the initial condition $\left(x_{0}, r_{0}\right)$. Corresponding to Theorem 7 we have

$$
\mathcal{F}\left(x_{0}, r_{0}\right)=\left.\frac{1}{2} \int_{0}^{2 \pi} M^{-1}\left(\begin{array}{c}
\left(2 d^{2}-x^{2}\right) \sin ^{2} \theta \\
\left(2 d^{2}-x^{2}\right) \cos \theta
\end{array}\right)\right|_{(8)} d \theta
$$

Then $F\left(x_{0}, r_{0}\right)=\left(g_{1}\left(x_{0}, r_{0}\right), g_{2}\left(x_{0}, r_{0}\right)\right)$ with

$$
g_{1}\left(x_{0}, r_{0}\right)=\frac{1}{4}\left(4 d^{2}-5 r_{0}^{2}-6 r_{0} x_{0}-2 x_{0}^{2}\right), \quad g_{2}\left(x_{0}, r_{0}\right)=\frac{1}{2} r_{0}\left(x_{0}+r_{0}\right) .
$$

We can check that $\mathcal{F}=0$ has a unique non-trivial solution $x_{0}=-2 d$ and $r_{0}=2 d$, and that $\left.\operatorname{det} D \mathcal{F}\left(x_{0}, r_{0}\right)\right|_{x_{0}=-2 d, r_{0}=2 d}=d^{2}$. Hence by Theorem 7 it follows that for any given $d>0$ and for $|\varepsilon|>0$ sufficiently small system $(7)$ has a periodic orbit $(x(\theta), r(\theta))$ of period $2 \pi$, such that $(x(0), r(0))$ approaches to $(-2 d, 2 d)$ as $\varepsilon \rightarrow 0$. We note that the eigenvalues of $\left.\operatorname{DF}\left(x_{0}, r_{0}\right)\right|_{x_{0}=-2 d, r_{0}=2 d}$ are $\pm \sqrt{-1} d$. This shows that the periodic orbit is linearly elliptic.

Going back to system (1) we get that for $c>0$ sufficiently small the Michelson system has a periodic orbit of period approximating $2 \pi: x(t)=$ $-2 c \cos t+o(c), y(t)=2 c \sin t+o(c)$ and $z(t)=2 c \cos t+o(c)$. This periodic orbit looks symmetric with respect to the involution $R$, because for $c=0$ it is symmetric.

\section{ACKNOWLEDGEMENTS}

The first author is partially supported by a MCYT/FEDER grant number MTM 2005-06098-C02-01 and by a CICYT grant number 2005SGR 00550. The second author is partially supported by NNSF of China grant 10671123 and NCET of China grant 050391. He thanks the Centre de Recerca Matemàtica for the hospitality and the financial support by the grant SAB2006-0098 (Ministerio de Educación y Ciencia, Spain). 


\section{REFERENCES}

[1] A. Buică, J.P. Françoise And J. Llibre, Periodic solutions of nonlinear periodic differential systems with a small parameter, Commun. Pure Appl. Anal. 6 (2007), 103-111.

[2] F. Dumortier, A. Ibáñez And H. Kokubu, Cocoon bifurcation in threedimensional reversible vector fields, Nonlinearity 19 (2006), 305-328.

[3] P. Kent And J. Elgin, Noose bifurcation of periodic orbits, Nonlinearity 4 (1991), $1045-1061$.

[4] H. Kokubu, D. Wilczak and P. Zgliczyński, Rigorous verification of cocoon bifurcations in the Michelson system, Nonlinearity 20 (2007), 2147-2174.

[5] J.S.W. LAmb And J.A.G. RoberTs, Time-reversal symmetry in dynamical systems: A survey, Physica D 2 (1998), 1-39.

[6] J.S.W. Lamb and M.A. Teixeira and K.N. Webster, Heteroclinic bifurcations near Hopf-zero bifurcation in reversible vector fields in $\mathbb{R}^{3}$, J. Diff. Eqns 219 (2005), $78-115$.

[7] Y-T. LAU, The 'cocoon' bifurcations in three-dimensional systems with two fixed points, Intern. J. Bifur. Chaos 2 (1992), 543-558.

[8] D. Michelson, Steady solutions for the Kuramoto-Sivashinsky equation, Physica D 19 (1986), 89-111.

[9] W.C. Troy, The existence of steady solutions of the Kuramoto-Sivashinsky equation, J. Differential Equations 82 (1989), 269-313.

[10] K.N. Webster, Bifurcations in reversible systems with application to the Michelson system, Ph.D. Thesis, 2005.

[11] K.N. Webster And J. Elgin, Asymptotic analysis of the Michelson system, Nonlinearity 16 (2003), 2149-2162.

${ }^{1}$ Departament de Matemàtiques, Universitat Autònoma de Barcelona, 08193 Bellaterra, Barcelona, Catalonia, Spain

E-mail address: jllibre@mat.uab.cat

2 Department of Mathematics, Shanghai Jiaotong University, Shanghai, 200240, P. R. CHINA

E-mail address: xzhang@sjtu.edu.cn 